\title{
Evolution of glomerular filtration rates and neutrophil gelatinase-associated lipocalin during treatment with direct acting antivirals
}

\author{
Alessio Strazzulla', Giuseppe Coppolino ${ }^{2}$, Giorgio Settimo Barreca ${ }^{3}$, Innocenza Gentile ${ }^{4}$, Laura Rivoli ${ }^{5}$, \\ Maria Concetta Postorino', Maria Mazzitelli', Giuseppe Greco', Chiara Costa', Vincenzo Pisani', Nadia Marascio ${ }^{3}$, \\ Mariadelina Simeoni ${ }^{5}$, Alfredo Focà ${ }^{3}$, Giorgio Fuiano ${ }^{5}$, Daniela Foti ${ }^{4}$, Elio Gulletta ${ }^{4}$ and Carlo Torti ${ }^{1}$ \\ 'Division of Infectious Diseases, Department of Medical and Surgical Sciences, Magna Graecia University, Catanzaro; ${ }^{2}$ Division of \\ Nephrology and Dialysis, Pugliese-Ciaccio Hospital, Catanzaro; ${ }^{3}$ Division of Microbiology, Department of Health Sciences, Magna Graecia \\ University, Catanzaro; ${ }^{4}$ Division of Clinical Pathology, Department of Health Sciences, Magna Graecia University, Catanzaro; ${ }^{5}$ Division \\ of Nephrology, Department of Medical and Surgical Sciences, Magna Graecia University, Catanzaro, Italy
}

Background/Aims: Correct renal function evaluation is based on estimated glomerular filtration rates (eGFR) and complementary renal damage biomarkers, such as neutrophil gelatinase associated lipocalin (NGAL). The aim of this study was to evaluate eGFR and NGAL modifications and renal impairment during treatment with a direct acting antiviral (DAA) for chronic hepatitis C virus (HCV) infection.

Methods: A retrospective cohort study evaluated eGFR modification during treatment with DAA. Subgroup analysis on serum NGAL was conducted in those receiving sofosbuvir/ledipasvir, with complete follow-up until week 12 after the end of treatment (FU-12).

Results: In the 102 enrolled patients, eGFR reduction was observed (from $86.22 \mathrm{~mL} / \mathrm{min}$ at baseline to $84.43 \mathrm{~mL} / \mathrm{min}$ at FU-12, $P=0.049$ ). Mean NGAL increased in 18 patients (from $121.89 \mathrm{ng} / \mathrm{mL}$ at baseline to $204.13 \mathrm{ng} / \mathrm{mL}$ at FU-12, $P=0.014$ ). At FU-12, 38.8\% (7/18) of patients had a plasmatic NGAL value higher than the normal range $(36-203 \mathrm{ng} / \mathrm{mL})$ compared with $11.1 \%(2 / 18)$ at baseline $\left(\chi^{2}=3,704 ; P=0.054\right)$. In contrast, eGFR did not change significantly over the follow-up in this subgroup.

Conclusions: In conclusion, compared to a negligible eGFR decline observed in the entire cohort analyzed, a significant NGAL increase was observed after HCV treatment with DAA in a small subgroup. This could reflect tubular damage during DAA treatment rather than glomerular injury. (Clin Mol Hepatol 2018;24:151-162)

Keywords: Hepatitis C virus; Inflammation; Kidney; Hepatitis; Antivirals

Study Highlights

For the first time, this study investigated the potential role of NGAL as marker of kidney injury during HCV treatment with interferon-free regimens. DAAs administration was associated with a significant serum NGAL increase not accompanied by a noteworthy eGFR decline. Compared to conventional renal markers, an additional role of NGAL could be proposed in early detecting DAA-induced nephrotoxicity mainly characterized by a tubular involvement.

\footnotetext{
Abbreviations:

ALT, alanine transaminase; APRI, aspartate transaminase to platelet ratio index; AST aspartate transaminase; BMI, body mass index; CKD, chronic kidney disease; DAA, directly acting antiviral; eGFR, estimated glomerular filtration rate; ELISA, enzyme-linked immunosorbent assay; FIB-4, fibrosis-4; HCV, hepatitis C virus; HIV human immunedeficiency virus; HRP, horseradish peroxidase; IFN, interferon; IRB, institutional review board; KDIGO, kidney disease improving global outcome; LDV, ledipasvir; NGAL, neutrophil gelatinase associated lipocalin; PI, prothease inhibitor; RBV, ribavirin; SOF, sofosbuvir; SVR, sustained virological response
}

\section{Corresponding author : Alessio Strazzulla}

Infectious Diseases Unit, Department of Medical and Surgical Sciences, Magna Graecia University, Viale Europa 88100, Catanzaro, Italy

Tel: +39-961-3647203, Fax: +39-961-3647544

E-mail: alessiostrazzulla@yahoo.it

http://orcid.org/0000-0003-0199-3449 


\section{INTRODUCTION}

Hepatitis $\mathrm{C}$ virus (HCV) treatments including directly acting antivirals (DAA) are highly effective. However, it is still unclear if DAA use in nephropathic patients is safe.' International guidelines recommend to avoid using sofosbuvir (SOF) in patients with an estimated glomerular filtration rate (eGFR) $\leq 30 \mathrm{~mL} / \mathrm{min}^{2,3}$ Although SOF pharmacokinetics needs to be better explored, it is well known that it mainly underrates renal clearance. However, recent literature data showed high rates of sustained virological response (SVR) without significant adverse events when SOF was prescribed at different doses (from $200 \mathrm{mg}$ daily to $400 \mathrm{mg}$ daily) in patients with severe renal impairment or on hemodialysis. ${ }^{4-8}$

Because kidney disease improving global outcome (KDIGO) guidelines suggest that available eGFR equations are biased in some conditions (e.g. high GFR, liver cirrhosis), they recommend to dose complementary biomarkers of renal damage beside serum creatinine (SCr). However, in the current clinical practice, renal safety during HCV treatment is routinely monitored only by creatinine and eGFR. ${ }^{9}$

Neutrophil gelatinase associated lipocalin (NGAL) is a novel kidney biomarker. It is a small glycoprotein secreted either by epithelial cells (liver, kidney, lungs) and blood cells (neutrophils, monocytes and macrophages), filtered in the glomerulus and reabsorbed by the proximal tubules. It can be dosed in blood and urine and it is used as early marker of acute kidney injury in many different settings, including immunosuppressive treatments (tacrolimus) and non-steroidal anti-inflammatory drug toxicity. ${ }^{10-12}$

In a recent study, we dosed plasmatic NGAL in a cohort of HCV infected patients and in a subgroup of patients treated with old generation DAA including regimens (pegylated-interferon [IFN] plus ribavirin [RBV] plus telaprevir or daclatasvir plus simeprevir). ${ }^{13}$ In our cohort, we observed a significant increase of plasma NGAL during the first 12 weeks of treatment. Because of our results and the limited knowledge on the renal safety of new generation DAA molecules (mainly SOF), we designed the present study assuming the following objectives: i) to evaluate the evolution of eGFR during therapy with the newer IFN-free regimens in a cohort of patients affected by chronic hepatitis $\mathrm{C}$ treated in our centre; ii) to evaluate the evolution of NGAL in a subgroup of patients treated with one of these regimens.

\section{MATERIALS AND METHODS}

\section{Recruitment of patients and data collection}

This retrospective cohort study was conducted at the Unit of Infectious and Tropical Diseases of the Mater Domini Teaching Hospital in Catanzaro (Italy) from January 31st, 2014 to October 31st, 2016. HCV positive patients who were prescribed treatments including a DAA agent were enrolled. The study was approved by the institutional review board (IRB) of "Mater Domini" Teaching Hospital (IRB No.: 1/CE_22/01/2014) and was conducted with respect of Declaration of Helsinki. All study participants provided written consent prior to study enrollment. To be recruited, patients had to have a scheduled end of treatment at least 12 weeks before 31st October 2016 (starting analysis date).

Exclusion criteria were: i) IFN including treatment; ii) co-infection with human immunodeficiency virus (HIV); iii) hemodialysis; iv) retreatment after an interval $<6$ months between the sequential treatment courses; v) treatment still ongoing or with $<12$ weeks of available follow-up at the completion of the study; vi) hepatitis B surface antigen positivity. ${ }^{14}$

Patients having all time-points available (baseline, end of treatment and week-12 of follow-up after the end of treatment [FU-12]) were included in the study of eGFR evolution while serum NGAL changes were evaluated in a subgroup of patients treated with SOF/ ledipasvir (LDV) for which a serum sample was stored in the laboratory of the Clinical Microbiology and Virology Unit of Mater Domini Teaching Hospital of Catanzaro (Italy). Medical history, physical examination and routinely laboratory exams were performed atbaseline, end of treatment and FU-12.

Chronic Kidney Disease Epidemiology Collaboration (CKD-Epi) equation $\left\{141 \times \min (\mathrm{SCr} / \mathrm{k}, 1)^{\alpha} \times \max (\mathrm{SCr} / \mathrm{k}, 1)^{-1.209} \times 0.993^{\mathrm{Age}} \times[1.018\right.$ if female or 1.159 if black]\} was used to calculate eGFR in which $\mathrm{SCr}$ is serum creatinine (in $\mathrm{mg} / \mathrm{dL}$ ), $\mathrm{k}$ is 0.7 for females and 0.9 for males, a is 0.329 for females and 0.411 for males, min is the minimum of $\mathrm{SCr} / \mathrm{k}$ or 1 , and max is the maximum of SCr/k or 1. CKD-Epi equation was preferred because is the most accurate formula for eGFR $\geq 60$ $\mathrm{mL} / \mathrm{min}^{9,15}$

Liver fibrosis was estimated at baseline by transient elastography and at each time-point either by Fibrosis-4 (FIB-4) score or aspartate transaminase (AST) to platelet ratio index (APRI). According to transient elastography, patients were considered cirrhotic when estimated liver stiffness was $\geq 14.5 \mathrm{kPa} .{ }^{16}$ For FIB- 4 calculation, the following formula was applied: age $\times$ AST/(platelets $\times$ Valanine transaminase [ALT]), in which AST and ALT were measured as IU/L, 
Alessio Strazzulla, et al. Evolution of NGAL and eGFR during HCV treatments

platelets as number $\times 10^{6} / \mu \mathrm{L}$ and age was reported in years. ${ }^{17}$ For APRI calculation, the following formula was used: ([AST/AST upper normal limit]/platelets) $\times 100$, in which AST was measured as IU/L, platelets as number $\times 10^{6} / \mu \mathrm{L}$ and AST upper normal limit was fixed at $35 \mathrm{IU} / \mathrm{L}^{18}$

\section{NGAL measurement}

NGAL was measured at baseline, end of treatment and FU-12. NGAL assay was performed on serum samples using the BioPorto Diagnostics Human NGAL enzyme linked immunosorbent assay (ELISA) Kit 036RUO (BioPorto Diagnostics A/S, Hellerup, Denmark), for the in vitro determination of human NGAL in body fluids (e.g. plasma, serum or urine). ${ }^{19}$ This is a sandwich ELISA performed in 96-microwells coated with a monoclonal antibody to human NGAL. The analyte was detected with another monoclonal NGAL antibody labeled with biotin and the assay was developed by horseradish peroxidase (HRP)-conjugated streptavidin, followed by the addition of a color-forming substrate.

Serum samples and NGAL controls were diluted 1:500 in a microwell plat. Each microwell was added of $100 \mu \mathrm{L}$ of undiluted calibrator and incubated for 60 minutes at room temperature. This step allowed NGAL binding to specific antibodies adsorbed to microwells. Unbound material was removed by using $300 \mu \mathrm{L}$ of wash solution for three washing cycles. Successively, 100 L biotinylated monoclonal detection antibody was added to each test well and incubated for 60 minutes at room temperature. After one more washing step, $100 \mu \mathrm{L}$ HRP-conjugated streptavidin was added to each test well and allowed to form a complex with the bound biotinylated antibody. Unbound conjugate was removed by washing. A colorforming peroxidase substrate containing tetramethylbenzidine was added to each test well. The bound HRP-streptavidin reacted with the substrate to generate a colored product. The enzymatic reaction was stopped chemically and the optical density was read at 450 $\mathrm{nm}$ in an ELISA reader. The absorbance was proportional to NGAL concentration in all tested samples. NGAL concentration in each sample was plotted on the validated calibration curve by a Triturus automatic analyzer. NGAL normal reference range (36-203 ng/mL) had been previously validated in our laboratory.

\section{Ranking of patients at baseline and statistical notes}

Patients were ranked at baseline as follows: i) KDIGO-CKD classification (stage 1 [eGFR $\geq 90 \mathrm{~mL} / \mathrm{min}$ ], stage 2 [eGFR 60-89 mL/ $\mathrm{min}$ ] and stages $3 a-5$ [eGFR $\leq 59 \mathrm{~mL} / \mathrm{min}]) ;$ ii) age ( $\geq 65$ years and
$<65$ years); iii) body mass index (BMI) (normal [18.5-24.9], overweight [25-29.9] and obese patients [ $\geq 30]$ ); iv) type of treatment (protease inhibitor [PI] including and non-PI including); v) use of ribavirin (yes and no); HCV-RNA ( $\geq 1,000,000 \mathrm{IU} / \mathrm{mL}$ and $<1,000,000$ $\mathrm{IU} / \mathrm{mL}$ ); cirrhosis (yes [transient elastography $\geq 14.5 \mathrm{kPa}$ or clinical cirrhosis] and no [liver transient elastography <14.5 kPa]).

Quantitative variables were presented in the text as mean values and analyzed through statistical analysis based on mean regression (Student's $t$-test) because we compared the same patients (without drop-out or drop-in) at the same time points. Main characteristics of the three populations (overall cohort, eGFR group and NGAL subgroup) were compared through Student's $t$-test (quantitative data: eGFR, age and FIB-4) and $\chi^{2}$ test (qualitative data: cirrhosis and gender), as appropriate.

Changes of eGFR were evaluated by comparing mean eGFR at the different time points (baseline vs. end of treatment, baseline vs. FU-12, end of treatment vs. FU-12) through paired Student's $t$-test in the overall population and in patients ranked at baseline by KDIGO-CKD classification, age, BMI, type of treatment, use of ribavirin, HCV-RNA levels, and cirrhosis. A regression analysis was performed to assess eGFR variations from baseline to the end of treatment ( $\triangle$ eGFR) adjusted for gender, age, use of ribavirin, basal eGFR and cirrhosis.

NGAL changes were evaluated by comparing mean serum NGAL at the different time points (baseline vs. end of treatment, baseline vs. FU-12, end of treatment vs. FU-12) through paired Student's ttest in the subgroup of patients included in NGAL study and in the same patients ranked at baseline by use of use of ribavirin and cirrhosis. In the same subgroup of patients, also eGFR changes were evaluated by comparing mean eGFR through Student's $t$-test.

In order to analyze serum NGAL trend in response to treatment, patients with serum NGAL value higher than the upper limit of normality range $(36-203 \mathrm{ng} / \mathrm{mL})$ were compared through $\chi^{2}$ test across established time points. Linear regression analysis was performed to assess correlation of serum NGAL at each time point with: i) eGFR; ii) FIB-4; iii) APRI. Statistical significance was set at $P \leq 0.05$.

\section{RESULTS}

\section{Study populations}

The study design is depicted in Figure 1. Overall, 149 patients received 162 treatments including DAAs (10 patients were being treated with 2 different regimens while 1 patients was treated with 


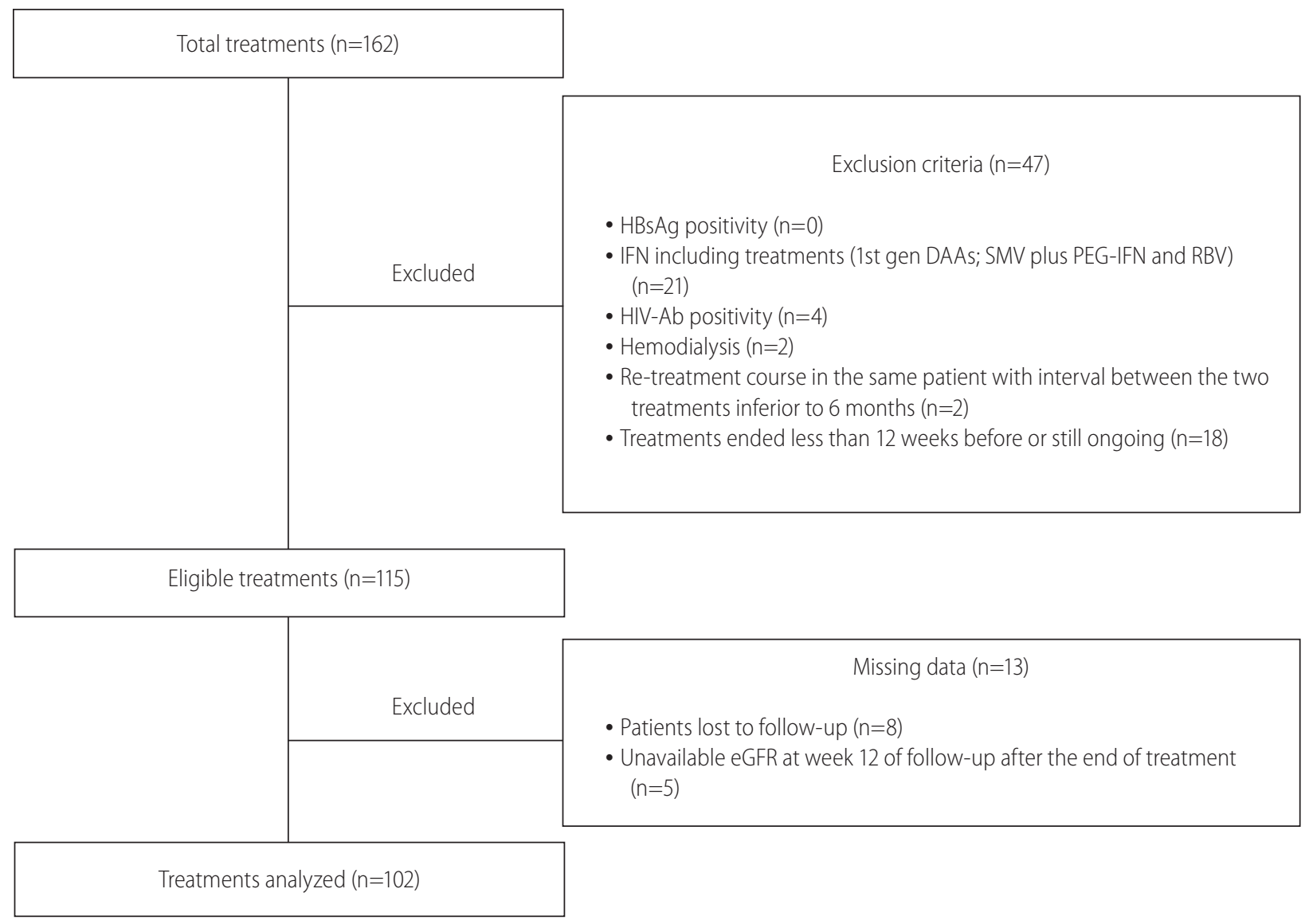

Figure 1. Flow chart of the study. The figure shows the flow chart of the study. Overall, 162 HCV treatment were completed by the end of the study. Of them, 47/162 were excluded because of exclusion criteria and 13 out of 115 eligible treatements were excluded for missing complete data at the end of the study. Finally 102/162 treatements were analyzed. HCV, hepatitis C virus; HBsAg, hepatitis B surface antigen; IFN, interferon; 1st gen DAA, first generation directly acting antivirals; SMV, simeprevir; PEG-IFN, pegylated interferon; RBV, ribavirin; HIV-Ab, human immunodeficiency virus antibodies; eGFR, estimated glomerular filtration rate.

four different regimens) from January 31st, 2014 to October 31st, 2016. According to the exclusion criteria, 47 treatments were removed (18 had a scheduled end of treatment less than 12 weeks before the starting analysis date or were still on course) and other 13 treatments were further excluded because of missing data (8 patients were lost to follow-up and 5 had not available eGFR at week 12 of follow-up after the end of treatment). Thus, eGFR changes were evaluated in 99 patients, accounting for 102 treatment courses. Serum NGAL changes were additionally evaluated only in 18 patients treated with SOF/LDV. Characteristics of patients at baseline of the first DAA including treatment course, characteristics and outcomes of treatments are summarized in Tables 1-3.

No statistically significant differences $(P>0.05)$ in gender distribution, percentage of cirrhosis, mean age, mean FIB-4 score and mean eGFR value at baseline were present among the overall pop- ulation (including all patients treated) and the two populations analyzed in the study (either for eGFR or NGAL analyses).

\section{Evaluation of eGFR and predictors}

Estimated GFR changes were analyzed in 99 patients, accounting for 102 treatments. Overall, eGFR reduction was observed from baseline to FU-12 (from $86.22 \mathrm{~mL} / \mathrm{min}$ to $84.3 \mathrm{ml} / \mathrm{min} ; P=0.049$ ) (Fig. 2). However, eGFR changes were not statistically different among patients ranked by age, BMI, type of regimen, HCV-RNA levels and cirrhosis at baseline. Interestingly, a statistically significant eGFR decrease (from $87.12 \mathrm{~mL} / \mathrm{min}$ at baseline to $84.4 \mathrm{~mL} /$ min at FU-12; $P=0.045$ ) was observed in patients on RBV including treatment. Conversely, patients treated with regimens not including RBV did not show significant eGFR changes (from 85.17 
Alessio Strazzulla, et al.

Evolution of NGAL and eGFR during HCV treatments

Table 1. Characteristics of the population at baseline

\begin{tabular}{|c|c|c|c|}
\hline Characteristics & $\begin{array}{l}\text { Overall population } \\
\qquad(n=149)\end{array}$ & $\begin{array}{l}\text { eGFR study population } \\
\qquad(n=99)\end{array}$ & $\begin{array}{l}\text { NGAL study population } \\
\qquad(\mathrm{n}=18)\end{array}$ \\
\hline \multicolumn{4}{|l|}{ Qualitative variables, n (\%) } \\
\hline \multicolumn{4}{|l|}{ Age } \\
\hline$\geq 65$ & $86(58)$ & $62(63)$ & $12(67)$ \\
\hline$<65$ & $63(42)$ & $37(37)$ & $6(33)$ \\
\hline \multicolumn{4}{|l|}{ Gender } \\
\hline Male & $84(56)$ & $54(55)$ & $11(61)$ \\
\hline Female & $65(44)$ & $45(45)$ & $7(39)$ \\
\hline \multicolumn{4}{|l|}{$\mathrm{BMI}$} \\
\hline Normal & $58(9)$ & $37(37)$ & $9(50)$ \\
\hline Overweight & $69(46)$ & $46(47)$ & $7(39)$ \\
\hline Obesity & $22(15)$ & $16(16)$ & $2(11)$ \\
\hline \multicolumn{4}{|l|}{ Cirrhosis } \\
\hline Yes & $77(48)$ & $51(52)$ & $9(50)$ \\
\hline No & $85(52)$ & $48(48)$ & $9(50)$ \\
\hline \multicolumn{4}{|l|}{ Child-Turcotte-Pugh } \\
\hline A & $147(99)$ & $99(100)$ & $18(0)$ \\
\hline B & $2(1)$ & $0(0)$ & $0(0)$ \\
\hline C & $0(0)$ & $0(0)$ & $0(0)$ \\
\hline \multicolumn{4}{|l|}{ HCV Genotype } \\
\hline $1 a$ & $6(4)$ & $3(3)$ & $1(6)$ \\
\hline $1 b$ & $124(76)$ & $72(73)$ & $17(94)$ \\
\hline 2 & $21(13)$ & $17(17)$ & $0(0)$ \\
\hline 3 & $5(3)$ & $2(2)$ & $0(0)$ \\
\hline 4 & $6(4)$ & $5(5)$ & $0(0)$ \\
\hline \multicolumn{4}{|l|}{ IFN experienced } \\
\hline Yes & $81(54)$ & $56(57)$ & $12(67)$ \\
\hline No & $68(46)$ & $43(43)$ & $6(33)$ \\
\hline \multicolumn{4}{|l|}{ Transplantation } \\
\hline Liver & $3(2)$ & $3(3)$ & $0(0)$ \\
\hline Kidney & $2(1)$ & $1(1)$ & $0(0)$ \\
\hline No & $144(97)$ & $95(96)$ & $18(100)$ \\
\hline \multicolumn{4}{|l|}{ HBV } \\
\hline $\mathrm{HBcAb}+\mathrm{HBsAg}-\mathrm{HBsAb}-$ & $24(16)$ & $17(17)$ & $2(11)$ \\
\hline $\mathrm{HBcAb}+\mathrm{HBsAg}+$ & $0(0)$ & $0(0)$ & $0(0)$ \\
\hline $\mathrm{HBcAb}-\mathrm{HBsAb+}$ & $14(9)$ & $9(9)$ & $1(5)$ \\
\hline $\mathrm{HBCAb}-\mathrm{HBsAb}-$ & $55(37)$ & $34(35)$ & $7(39)$ \\
\hline $\mathrm{HBcAb}+\mathrm{HBsAb}+$ & $16(11)$ & $8(8)$ & $3(17)$ \\
\hline Data not available & $40(27)$ & $31(31)$ & $5(28)$ \\
\hline \multicolumn{4}{|l|}{ HIV } \\
\hline $\mathrm{HIV}-\mathrm{Ab}+$ & $4(3)$ & $0(0)$ & $0(0)$ \\
\hline HIV-Ab- & $145(97)$ & $99(100)$ & $18(100)$ \\
\hline
\end{tabular}


Table 1. Continued

\begin{tabular}{|c|c|c|c|}
\hline Characteristics & $\begin{array}{l}\text { Overall population } \\
\qquad(n=149)\end{array}$ & $\begin{array}{l}\text { eGFR study population } \\
\qquad(n=99)\end{array}$ & $\begin{array}{l}\text { NGAL study population } \\
\qquad(\mathrm{n}=18)\end{array}$ \\
\hline \multicolumn{4}{|l|}{ Diabetes } \\
\hline Yes & $34(23)$ & $24(24)$ & $5(28)$ \\
\hline No & $115(77)$ & $75(76)$ & $13(72)$ \\
\hline \multicolumn{4}{|l|}{ Hypertension } \\
\hline Yes & $68(46)$ & $54(55)$ & $9(50)$ \\
\hline No & $81(54)$ & $45(45)$ & $9(50)$ \\
\hline \multicolumn{4}{|l|}{ Use of NSAIDs } \\
\hline Yes & $9(6)$ & $7(7)$ & $1(6)$ \\
\hline No & $140(94)$ & $92(93)$ & $17(94)$ \\
\hline \multicolumn{4}{|l|}{ CKD classification } \\
\hline Stage 1 & $84(52)$ & $54(55)$ & $9(50)$ \\
\hline Stage 2 & $62(38)$ & $37(37)$ & $7(39)$ \\
\hline Stage $3 a$ & $9(5)$ & $4(4)$ & $2(11)$ \\
\hline Stage $3 b$ & $6(4)$ & $4(4)$ & $0(0)$ \\
\hline Stage 4 & $0(0)$ & $0(0)$ & $0(0)$ \\
\hline Stage 5 & $1(1)$ & $0(0)$ & $0(0)$ \\
\hline \multicolumn{4}{|l|}{ Hemodialysis } \\
\hline Yes & $2(1)$ & $0(0)$ & $0(0)$ \\
\hline No & 147 (99) & $99(100)$ & $18(100)$ \\
\hline \multicolumn{4}{|l|}{ Quantitative variables, mean (SD) } \\
\hline Age (years) & $64.1(10.5)$ & $65.7(8.9)$ & $66.7(8.1)$ \\
\hline BMI & $26.4(4.6)$ & $26.9(5.1)$ & $25.9(3.4)$ \\
\hline HCV-RNA (IU/mL) & $3,105,702(4,435,652)$ & $3,551,044(5,001,105)$ & $1,132,736(1,520,590)$ \\
\hline Hemoglobin (g/dL) & $14.3(2.0)$ & $14.2(2.1)$ & $14.0(2.3)$ \\
\hline Platelets $\left(\mathrm{n} \times 10^{3} / \mu \mathrm{L}\right)$ & $164(71)$ & $158(68)$ & $160(73)$ \\
\hline Leukocytes (cell/ $\mu \mathrm{L})$ & $6,173(2,062)$ & $5,885(1,892)$ & $5,781(2,11)$ \\
\hline INR & $1.1(0.1)$ & $1.1(0.1)$ & $1.1(0.1)$ \\
\hline Glucose (mg/dL) & $117(39)$ & $117(42)$ & $122(67)$ \\
\hline Cholesterol (mg/dL) & $155(38)$ & $154(41)$ & $151(46)$ \\
\hline Triglyceride (mg/dL) & $118(64)$ & $106(51)$ & $98(45)$ \\
\hline AST (IU/L) & $62(42)$ & $60(41)$ & $48(23)$ \\
\hline ALT (IU/L) & $74(69)$ & $65(50)$ & $62(62)$ \\
\hline Alkaline phosphatase (IU/L) & $106(65)$ & $110(71)$ & $102(59)$ \\
\hline Y-glutamyltransferase (IU/L) & $76(74)$ & $67(55)$ & $54(36)$ \\
\hline Total bilirubin (mg/dL) & $0.8(0.6)$ & $0.8(0.7)$ & $0.7(0.3)$ \\
\hline Albumin (g/dL) & $4.1(0.5)$ & $4.1(0.4)$ & $4.0(0.5)$ \\
\hline FIB-4 & $3.9(3.6)$ & $4.2(3.9)$ & $2.8(2.4)$ \\
\hline APRI & $1.5(1.5)$ & $1.5(1.5)$ & $1.3(1.3)$ \\
\hline Creatinine (mg/dL) & $0.9(0.8)$ & $0.8(0.2)$ & $0.8(0.2)$ \\
\hline Urea $(\mathrm{g} / \mathrm{dL})$ & $41(22)$ & $40(16)$ & $44(19)$ \\
\hline eGFR (mL/min) & $86(19)$ & $86(17)$ & $83(18)$ \\
\hline
\end{tabular}

Values are presented as $\mathrm{n}(\%)$ or mean (SD) unless otherwise indicated.

eGFR, estimated glomerular filtration rate; NGAL, neutrophil gelatinase associated lipocalin; BMI, body mass index; HCV, hepatitis C virus; IFN, interferon; HBCAb, hepatitis B core antibody; HBsAg, hepatitis B surface antigen; HBV, hepatitis B virus; HBsAb, hepatitis B surface antibody; HIV, human immunodeficiency virus; NSAIDs, non-steroidal anti-inflammatory drugs; CKD, chronic kidney disease; SD, standard deviation; RNA, ribonucleic acid; INR, International normalized ratio; AST, aspartate aminotrasferase; ALT, alanine aminotransferase; FIB-4, Fibrosis-4; APRI, AST to platelet ratio index. 
Alessio Strazzulla, et al.

Evolution of NGAL and eGFR during HCV treatments

Table 2. Hepatitis C virus treatment characteristics and outcomes

\begin{tabular}{|c|c|c|c|}
\hline Characteristics & $\begin{array}{l}\text { Overall treatments } \\
\qquad(n=162)\end{array}$ & $\begin{array}{l}\text { eGFR study treatments } \\
\qquad(n=102)\end{array}$ & $\begin{array}{l}\text { NGAL study treatments } \\
\qquad(n=18)\end{array}$ \\
\hline \multicolumn{4}{|l|}{ DAAs } \\
\hline SOF & $13(8)$ & $12(12)$ & $0(0)$ \\
\hline SOF+LDV & $57(35)$ & $38(37)$ & $18(100)$ \\
\hline SOF+DCV & $27(17)$ & $16(16)$ & $0(0)$ \\
\hline SOF+SMV & $37(23)$ & $31(30)$ & $0(0)$ \\
\hline $\mathrm{SMV}+\mathrm{DCV}$ & $2(1)$ & $2(2)$ & $0(0)$ \\
\hline SOF+SMV+DCV & $1(1)$ & $0(0)$ & $0(0)$ \\
\hline $\mathrm{PTV} / \mathrm{OBV} / \mathrm{r}+\mathrm{DSB}$ & $4(2)$ & $3(3)$ & $0(0)$ \\
\hline IFN+SMV & $1(1)$ & $0(0)$ & $0(0)$ \\
\hline IFN+Telaprevir & $16(10)$ & $0(0)$ & $0(0)$ \\
\hline IFN+Boceprevir & $4(2)$ & $0(0)$ & $0(0)$ \\
\hline \multicolumn{4}{|l|}{ Ribavirin } \\
\hline Yes & $94(58)$ & $55(54)$ & $6(33)$ \\
\hline No & $68(42)$ & $47(46)$ & $12(67)$ \\
\hline \multicolumn{4}{|l|}{ Scheduled duration } \\
\hline 12 weeks & $81(50)$ & $61(60)$ & $6(33)$ \\
\hline 24 weeks & $64(40)$ & $41(40)$ & $12(67)$ \\
\hline 48 weeks (1st Gen. DAAs) & $17(10)$ & $0(0)$ & $0(0)$ \\
\hline \multicolumn{4}{|l|}{ Outcomes } \\
\hline SVR 12 & $123(76)$ & $97(95)$ & $18(100)$ \\
\hline Data not available & $18(11)$ & $0(0)$ & $0(0)$ \\
\hline Lost to follow-up & $8(5)$ & $0(0)$ & $0(0)$ \\
\hline Failure & $13(8)$ & $5(5)$ & $0(0)$ \\
\hline Failure with 1st Gen. DAAs & $8(5)$ & $0(0)$ & $0(0)$ \\
\hline Failure with 2nd Gen. DAAs & $5(3)$ & $0(0)$ & $0(0)$ \\
\hline
\end{tabular}

Values are presented as $n(\%)$.

eGFR, estimated glomerular filtration rate; NGAL, neutrophil gelatinase associated lipocalin; DAAs, directly acting antivirals; SOF, sofosbuvir; LDV, ledipasvir; DCV, daclatasvir; SMV, simeprevir; PTV, paritaprevir; OVB, ombitasvir; r, ritonavir; DSB, dasabuvir; IFN, interferon; Gen. DAA, generation directly acting antivirals; SVR, sustained virological response.

$\mathrm{mL} / \mathrm{min}$ at baseline to $83.95 \mathrm{~mL} / \mathrm{min}$ at FU-12; $P=0.822)$. According to patients ranking by KDIGO-CKD classification, eGFR was significantly reduced by the treatment only in patients with KDIGO-CKD stage 1 (from $97.99 \mathrm{~mL} / \mathrm{min}$ at baseline to $94.46 \mathrm{~mL} / \mathrm{min}$ at the end of treatment $[P<0.001]$ and $93.83 \mathrm{~mL} / \mathrm{min}$ at FU-12 $[P<$ $0.001])$. No statistical significant changes of eGFR were observed in patients ranked as KDIGO-CKD stage 2 and 3a-3b. At the multiple linear regression analysis, eGFR value at baseline (coeff. 0.1; SE $0.04 ; P=0.013$ ) was the only variable found to be predictive of $\triangle \mathrm{eGFR}$ from baseline to the end of treatment. No correlations were found between $\triangle \mathrm{eGFR}$ from baseline to end of treatment and age, use of RBV, cirrhosis and gender ( $P>0.05)$. No patients experienced acute kidney injury (increase in serum creatinine of $>50 \%$ above the baseline value or an increase of $\geq 0.3 \mathrm{mg} / \mathrm{dL}$ during last 48 hours).

\section{Evaluation of NGAL}

Serum NGAL changes were evaluated in 18 patients and a significant increase was observed. (from $121.89 \mathrm{ng} / \mathrm{mL}$ at baseline to $204.13 \mathrm{ng} / \mathrm{mL}$ at FU-12; $P=0.014$ ) (Fig. 3A). Notably, at FU-12, 38.8\% (7/18) patients had a plasmatic NGAL value higher than the normal range $(36-203 \mathrm{ng} / \mathrm{mL})$ compared with $11.1 \%(2 / 18)$ patients at 
Volume_24 Number_2 June 2018

Table 3. Patient characteristics at baseline according to the use of RBV

\begin{tabular}{|c|c|c|c|c|c|c|}
\hline \multirow{2}{*}{ Characteristics } & \multicolumn{2}{|c|}{ Overall population $(n=149)$} & \multicolumn{2}{|c|}{ eGFR study population $(n=99)$} & \multicolumn{2}{|c|}{ NGAL study population $(n=18)$} \\
\hline & RBV Yes & RBV No & RBV Yes & RBV No & RBV Yes & RBV No \\
\hline \multicolumn{7}{|c|}{ Qualitative variables, n (\%) } \\
\hline \multicolumn{7}{|l|}{ Age } \\
\hline$\geq 65$ & $48(55)$ & $40(65)$ & $32(60)$ & $28(61)$ & $2(33)$ & $10(83)$ \\
\hline$<65$ & $39(45)$ & $22(35)$ & $21(40)$ & $18(39)$ & $4(67)$ & $2(17)$ \\
\hline \multicolumn{7}{|l|}{ Cirrhosis } \\
\hline Yes & $39(45)$ & $31(50)$ & $27(51)$ & $24(52)$ & $2(33)$ & $8(67)$ \\
\hline No & $48(55)$ & $31(50)$ & $26(49)$ & $22(48)$ & $4(67)$ & $4(33)$ \\
\hline \multicolumn{7}{|l|}{ Child-Turcotte-Pugh } \\
\hline A & $87(100)$ & $60(50)$ & $53(100)$ & $46(100)$ & $6(100)$ & $12(100)$ \\
\hline B & $0(0)$ & $2(1)$ & $0(0)$ & $0(0)$ & $0(0)$ & $0(0)$ \\
\hline C & $0(0)$ & $0(0)$ & $0(0)$ & $0(0)$ & $0(0)$ & $0(0)$ \\
\hline \multicolumn{7}{|l|}{ HCV Genotype } \\
\hline $1 \mathrm{a}$ & $3(3)$ & $3(5)$ & $2(4)$ & $1(85)$ & $1(17)$ & $0(0)$ \\
\hline $1 \mathrm{~b}$ & $61(71)$ & $49(79)$ & $33(62)$ & $39(13)$ & $5(83)$ & $12(100)$ \\
\hline 2 & $12(14)$ & $9(14)$ & $11(21)$ & $6(2)$ & $0(0)$ & $0(0)$ \\
\hline 3 & $4(5)$ & $1(2)$ & $2(4)$ & $0(0)$ & $0(0)$ & $0(0)$ \\
\hline 4 & $6(7)$ & $0(0)$ & $5(9)$ & $0(0)$ & $0(0)$ & $0(0)$ \\
\hline \multicolumn{7}{|l|}{ IFN experienced } \\
\hline Yes & $54(62)$ & $27(44)$ & $34(64)$ & $22(48)$ & $5(83)$ & $7(58)$ \\
\hline No & $33(38)$ & $35(56)$ & $19(36)$ & $24(52)$ & $1(17)$ & $5(42)$ \\
\hline \multicolumn{7}{|l|}{ Transplantation } \\
\hline Liver & $85(98)$ & $59(95)$ & $52(98)$ & $44(96)$ & $6(100)$ & $12(100)$ \\
\hline Kidney & $1(1)$ & $2(3)$ & $1(2)$ & $2(4)$ & $0(0)$ & $0(0)$ \\
\hline No & $1(1)$ & $1(2)$ & $0(0)$ & $0(0)$ & $0(0)$ & $0(0)$ \\
\hline \multicolumn{7}{|l|}{ CKD classification } \\
\hline Stage 1 & $50(57)$ & $26(42)$ & $31(58)$ & $23(50)$ & $3(50)$ & $6(50)$ \\
\hline Stage 2 & $31(36)$ & $26(42)$ & $18(34)$ & $19(41)$ & $2(33)$ & $5(42)$ \\
\hline Stage 3a & $4(5)$ & $5(8)$ & $3(6)$ & $1(2)$ & $1(17)$ & $1(8)$ \\
\hline Stage 3b & $2(2)$ & $4(6)$ & $1(2)$ & $3(7)$ & $0(0)$ & $0(0)$ \\
\hline Stage 4 & $0(0)$ & $0(0)$ & $0(0)$ & $0(0)$ & $0(0)$ & $0(0)$ \\
\hline Stage 5 & $0(0)$ & $1(2)$ & $0(0)$ & $0(0)$ & $0(0)$ & $0(0)$ \\
\hline \multicolumn{7}{|c|}{ Quantitative variables, mean (SD) } \\
\hline Age (years) & $63.1(10.2)$ & $65.6(11.6)$ & $66.0(8.4)$ & $65.7(9.8)$ & $63.0(9.9)$ & $68.7(6.7)$ \\
\hline HCV-RNA (IU/mL) & $\begin{array}{l}2,706,599 \\
(3,551,031)\end{array}$ & $\begin{array}{c}3,687,451 \\
(5,549,958)\end{array}$ & $\begin{array}{c}3,134,696 \\
(4,291,693)\end{array}$ & $\begin{array}{c}3,979,707 \\
(5,669,199)\end{array}$ & $\begin{array}{c}934,385 \\
(181,8095)\end{array}$ & $\begin{array}{c}1,231,911 \\
(1,427,836)\end{array}$ \\
\hline Hemoglobin (g/dL) & $14.9(1.8)$ & $13.4(1.9)$ & $14.9(2.1)$ & $13.5(1.9)$ & $14.8(2.2)$ & $13.7(2.4)$ \\
\hline Platelets $\left(\mathrm{n} \times 10^{3} / \mu \mathrm{L}\right)$ & $157.34(60.30)$ & $180.75(86.65)$ & $154.23(67.72)$ & $164.76(70.20)$ & $134.17(76.32)$ & $174.00(72.20)$ \\
\hline
\end{tabular}


Alessio Strazzulla, et al. Evolution of NGAL and eGFR during HCV treatments

Table 3. Continued

\begin{tabular}{|c|c|c|c|c|c|c|}
\hline \multirow{2}{*}{ Characteristics } & \multicolumn{2}{|c|}{ Overall population $(n=149)$} & \multicolumn{2}{|c|}{ eGFR study population $(n=99)$} & \multicolumn{2}{|c|}{ NGAL study population $(n=18)$} \\
\hline & RBV Yes & RBV No & RBV Yes & RBV No & RBV Yes & RBV No \\
\hline Leukocytes (cell/ $\mu \mathrm{L})$ & $6,486(2,044)$ & $5,858(2,155)$ & $6,046(1,931)$ & $5,823(1,823)$ & $4,995(2,093)$ & $6,175(2,094)$ \\
\hline AST (IU/L) & $67(38)$ & $54(49)$ & $68(38)$ & $53(44)$ & $57(31)$ & $44(19)$ \\
\hline ALT (IU/L) & $80(59)$ & $68(86)$ & $74(47)$ & $57(53)$ & $62(44)$ & $62(72)$ \\
\hline Total bilirubin (mg/dL) & $0.8(0.6)$ & $0.9(0.7)$ & $0.9(0.7)$ & $0.8(0.7)$ & $0.6(0.3)$ & $0.7(0.3)$ \\
\hline Albumin (g/dL) & $4.2(0.5)$ & $4.0(0.4)$ & $4.2(0.4)$ & $4.0(0.4)$ & $4.1(0.6)$ & $3.9(0.4)$ \\
\hline $\mathrm{FIB}-4$ & $4.0(3.3)$ & $3.8(4.4)$ & $2.8(2.4)$ & $2.8(3.2)$ & $5.3(5.7)$ & $3.3(2.6)$ \\
\hline APRI & $1.6(1.5)$ & $1.2(1.6)$ & $1.7(1.5)$ & $1.3(1.5)$ & $1.9(1.9)$ & $0.9(0.7)$ \\
\hline Creatinine (mg/dL) & $0.8(0.3)$ & $1.0(1.2)$ & $0.8(0.2)$ & $0.8(0.2)$ & $0.9(0.2)$ & $0.8(0.2)$ \\
\hline Urea (mg/dL) & $38.7(15.5)$ & $48.6(29.7)$ & $37.4(13.8)$ & $43.4(17.8)$ & $35.4(9.2)$ & $48.5(22.5)$ \\
\hline eGFR (mL/min) & $88.9(17.0)$ & $80.5(23.3)$ & $87.1(16.7)$ & 84.5 (19.2) & $83.3(22.4)$ & $82.5(17.4)$ \\
\hline
\end{tabular}

Values are presented as $n(\%)$ or mean (SD) unless otherwise indicated.

RBV, ribavirin; eGFR, estimated glomerular filtration rate; NGAL, neutrophil gelatinase associated lipocalin; HCV, hepatitis C virus; IFN, interferon; CKD, chronic kidney disease; AST, aspartate transaminase; ALT, alanine transaminase; FIB-4, Fibrosis-4; APRI, AST to platelet ratio index.

baseline $\left(\chi^{2}=3,704 ; P=0.054\right)$. By ranking patients per use of RBV, no statistically significant differences in serum NGAL were found between groups, although a serum NGAL increase was transversally observed (RBV group: from $69.33 \mathrm{ng} / \mathrm{mL}$ at baseline to 161.39 $\mathrm{ng} / \mathrm{mL}$ at FU-12, $P=0.075$; no RBV group: from $148.16 \mathrm{ng} / \mathrm{mL}$ at baseline to $225.50 \mathrm{ng} / \mathrm{mL}$ at FU-12, $P=0.301)$. In contrast, noncirrhotic patients showed a significant increase in serum NGAL from baseline to FU-12 (from $85.33 \mathrm{ng} / \mathrm{ml}$ to $187.85 \mathrm{ng} / \mathrm{mL}, P=0.021$ ). No statistically significant differences in $\mathrm{GGF}$ evolution were found (Fig. 3B).

\section{DISCUSSION}

Concerns on renal safety may represent a limitation to a wide use of DAAs in HCV patients, despite the proven efficacy of this class of drugs. Furthermore, the reported unreliability of conventional markers of renal function in patients with liver cirrhosis can contribute to discourage DAA prescription. ${ }^{9}$ In our cohort study, we retrospectively investigated the impact of new generation DAA agents on renal function. In order to early detect possible signs of DAA-related nephrotoxicity, we evaluated not only eGFR changes in response to treatment but we also explored NGAL, a new promising biomarker of tubular impairment.

The most interesting result of our study is the significant serum
NGAL increase observed during treatment with SOF/LDV and in absence of a parallel worsening of eGFR. Our observation suggests that serum NGAL could have an additional role in predicting DAArelated nephrotoxicity, compared with conventional kidney markers. Moreover, in several previous studies, NGAL and creatinine provided complementary information on renal function impairment in several circumstances. ${ }^{10-12,20}$

NGAL is mainly a tubular biomarker. Indeed, many authors demonstrated that its urinary concentration correlates with the presence of tubular proteinuria and it is supposed that its increase could predict the presence of proteinuria. ${ }^{21-23}$ Moreover, its increase is not necessary accompanied by a glomerular impairment. Also, recent studies reported a good drug safety in patients with severe glomerular impairment treated with SOF. ${ }^{24,25}$ Even though one of the main limitations of our retrospective study is unavailability of other reliable biomarkers of renal impairment, such as proteinuria and cystatin $\mathrm{C}^{26}$, we might speculate that the increase of NGAL observed in our study was mainly related to a tubular rather than a glomerular impairment. In line with this interpretation, we did not find any significant eGFR decrease in the same patients.

On the other hand, it is important to consider that NGAL levels might raise not only as a consequence of renal damage but also of inflammation ${ }^{27,28}$ and this aspect limits NGAL reliability in some conditions. Indeed, although a favorable impact on renal function could be expected as a consequence of the eradication of HCV from glo- 


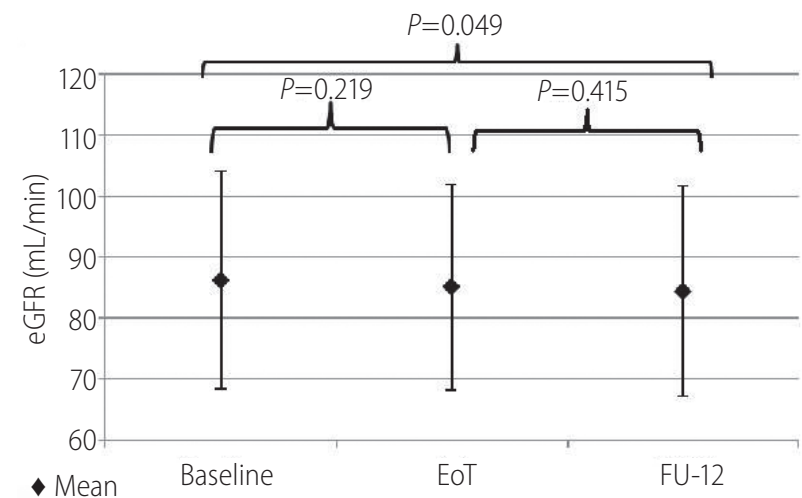

Figure 2. Evolution of estimated glomerular filtration rate (eGFR) in patients included in the eGFR evaluation study ( $N=102)$. The figure shows that the mean value of eGFR slightly reduced from baseline to week 12 of follow up (from $86.22 \mathrm{~mL} / \mathrm{min}$ to $84.3 \mathrm{~mL} / \mathrm{min} ; P=0.049$ ).
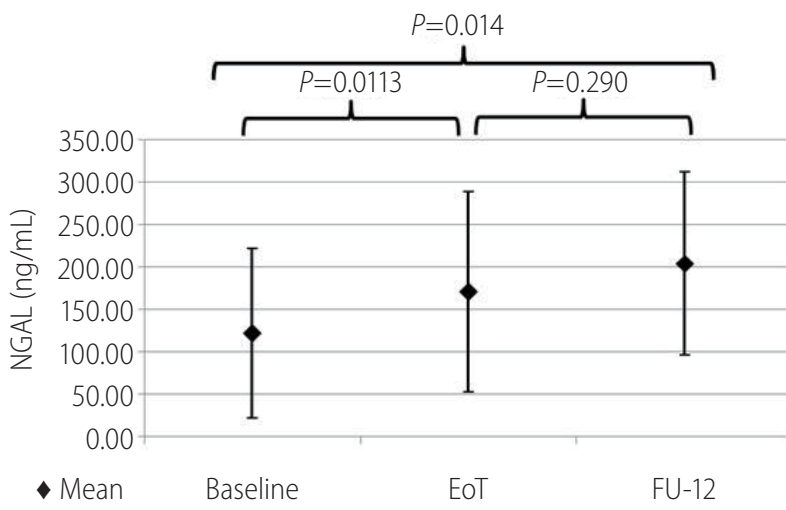

A

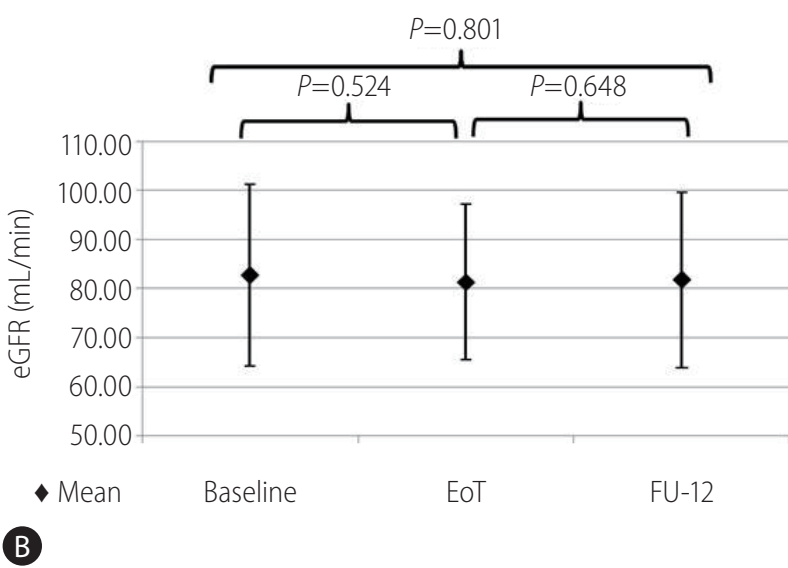

Figure 3. Evolution of serum neutrophil gelatinase associated lipocalin (NGAL) (A) and estimated glomerular filtration rate (eGFR) (B) in patients included in the NGAL evolution study $(\mathrm{N}=18)$. The figure shows that the mean value of NGAL increased significantly from baseline to week 12 of follow up (from $121.89 \mathrm{ng} / \mathrm{mL}$ at baseline to $204.13 \mathrm{ng} / \mathrm{mL}$ at FU-12; $P=0.014)(A)$ while a significantly change was not observed in mean eGFR value (B). merular and tubular cell $\mathrm{s}^{29}$, it was reported that HCV eradication during DAA treatment is accompanied by deregulation of inflammation response ${ }^{30,31}$ and possibly induces a paradox NGAL increase in absence of renal impairment.

This study reported a statistically significant but not clinically relevant eGFR reduction in the overall population analyzed and in patients with normal eGFR at baseline (KDIGO-CKD stage 1). However, multivariate analysis revealed that only higher eGFR at baseline resulted to be predictive of eGFR decrease during treatment with DAAs, suggesting that such decrease was simply due to a regression to the mean effect, rather than being a marker of a glomerular damage.

The modest though statistical significant eGFR decrease observed in patients treated with RBV is difficult to explain. At best of our knowledge, in fact, no data on RBV nephrotoxicity are available. ${ }^{32}$ However, considering that no patients experienced a clinically relevant renal impairment, we would conclude for a renal safety of DAAs with or without ribavirin (at least when glomerular function is considered) in patients with basal eGFR $\geq 60 \mathrm{mg} / \mathrm{mL}$.

Our results are limited by the retrospective design of the study and by different sizes of the study groups. Indeed, it is not possible to exclude that either the absence of eGFR reduction and the NGAL increase observed in the NGAL group were merely due to the small sample size. Also, because serum NGAL levels seem to be directly correlated with urinary NGAL levels, a urinary NGAL/ serum NGAL ratio may be a better marker of renal impairment. Future studies should be conducted to evaluate whether consideration of urinary NGAL would improve sensitivity and clinical prediction compared with the mere consideration of serum NGAL as performed in the present study.

In conclusion, our study demonstrated that DAAs administration is associated with a significant increase of serum NGAL not accompanied by a noteworthy eGFR decline. This relevant result might be expression of an additional role of NGAL, compared to conventional renal markers, in early detecting DAA-induced nephrotoxicity mainly characterized by a tubular involvement. However, we acknowledge that larger studies with a prospective design would be needed to confirm our results. In particular, both NGAL and eGFR (and possibly cystatin C) changes should be evaluated in HCV patients with severe renal disease (KDIGO-CKD classification stages 4 and 5) treated with DAAs and with the same duration of treatments. Also, it should be clarified whether the increase of serum NGAL was related to a tubular damage or other causes, such as deregulation of inflammatory process upon HCV eradication. 
Alessio Strazzulla, et al. Evolution of NGAL and eGFR during HCV treatments

\section{Author contributions}

Strazzulla A drafted the article, Coppolino G participated in design and oversight of the study; Barreca GS performed microbiological analysis, Gentile I made NGAL measurements, Rivoli L drafted the article and contributed to statistical analysis, Postorino MC performed statistical analysis, Mazzitelli M was involved in data collection, Greco $\mathrm{G}$ was involved in data collection, Costa $\mathrm{C}$ was involved in data collection, Pisani $V$ was involved in data collection, Marascio N was involved in data collection, Simeoni M drafted the article, Focà A participated in oversight the study, Fuiano $G$ participated in oversight the study, Foti D participated in oversight the study, Gulletta E participated in oversight the study, Torti C participated in oversight the study and drafted the article.

\section{Conflicts of Interest}

The authors have no conflicts to disclose.

\section{REFERENCES}

1. Mendizabal M, Reddy KR. Chronic hepatitis C and chronic kidney disease: advances, limitations and unchartered territories. J Viral Hepat 2017;24:442-453.

2. American Association for the Study of the Liver Diseases and the Infectious Diseases Society of America (AASLD-IDSA). Recommendations for testing, managing, and treating hepatitis C. AASLD-IDSA website, <https://www.hcvguidelines.org/>. Accessed 2017.02.13

3. European Association for the Study of the Liver. EASL Recommendations on Treatment of Hepatitis C 2016. J Hepatol 2017;66:153-194.

4. Mücke MM, Mücke VT, Lange CM, Zeuzem S. Special populations: treating hepatitis $C$ in patients with decompensated cirrhosis and/or advanced renal impairment. Liver Int 2017;37 Suppl 1:19-25.

5. Nazario HE, Ndungu M, Modi AA. Sofosbuvir and simeprevir in hepatitis $C$ genotype 1-patients with end-stage renal disease on haemodialysis or GFR <30 ml/min. Liver Int 2016;36:798-801.

6. Bhamidimarri KR, Czul F, Peyton A, Levy C, Hernandez M, Jeffers $L$, et al. Safety, efficacy and tolerability of half-dose sofosbuvir plus simeprevir in treatment of Hepatitis $C$ in patients with end stage renal disease. J Hepatol 2015;63:763-765.

7. Hundemer GL, Sise ME, Wisocky J, Ufere N, Friedman LS, Corey $\mathrm{KE}$, et al. Use of sofosbuvir-based direct-acting antiviral therapy for hepatitis $C$ viral infection in patients with severe renal insufficiency. Infect Dis (Lond) 2015;47:924-929.

8. Saxena V, Koraishy FM, Sise ME, Lim JK, Schmidt M, Chung RT, et al, Safety and efficacy of sofosbuvir-containing regimens in hepatitis C- infected patients with impaired renal function. Liver Int 2016;36:807816.

9. Levin A, Stevens PE, Bilous RW, Coresh J, De Francisco AL, De Jong $P E$, et al. Kidney Disease: Improving Global Outcomes (KDIGO) CKD Work Group. KDIGO 2012 clinical practice guideline for the evaluation and management of chronic kidney disease. Kidney Int Suppl 2013;3:1-150.

10. Firu SG, Streba CT, Firu D, Tache DE, Rogoveanu I. Neutrophil Gelatinase Associated Lipocalin (NGAL)-a biomarker of renal dysfunction in patients with liver cirrhosis: Do we have enough proof? J Med Life 2015;8 Spec Issue:15-20.

11. Tsuchimoto A, Shinke $H$, Uesugi $M$, Kikuchi M, Hashimoto $E$, Sato $T$, et al. Urinary neutrophil gelatinase-associated lipocalin: a useful biomarker for tacrolimus-induced acute kidney injury in liver transplant patients. PLoS One 2014;9:e110527.

12. Elia C, Graupera I, Barreto R, Solà E, Moreira R, Huelin P, et al. Severe acute kidney injury associated with non-steroidal anti-inflammatory drugs in cirrhosis: a case-control study. J Hepatol 2015;63:593-600.

13. Strazzulla A, Coppolino G, Di Fatta C, Giancotti F, D'Onofrio G, Postorino $M C$, et al. Is neutrophil gelatinase associated lipocalin useful in hepatitis C virus infection? World J Hepatol 2016;8:815-824.

14. Coppolino G, Simeoni M, Summaria C, Postorino MC, Rivoli L, Strazzulla $A$, et al. The case of chronic hepatitis B treatment with tenofovir: an update for nephrologists. J Nephrol 2015;28:393-402.

15. Cianfrone P, Simeoni M, Comi N, Piraina V, Talarico R, Cerantonio $A$, et al. How to improve duration and efficiency of the antiproteinuric response to Ramipril: RamiPROT-a prospective cohort study. J Nephrol 2017;30:95-102.

16. Ziol M, Handra-Luca A, Kettaneh A, Christidis C, Mal F, Kazemi F, et al. Noninvasive assessment of liver fibrosis by measurement of stiffness in patients with chronic hepatitis C. Hepatology 2005;41:48-54.

17. Vallet-Pichard A, Mallet V, Nalpas B, Verkarre V, Nalpas A, DhalluinVenier $V$, et al. FIB-4: an inexpensive and accurate marker of fibrosis in HCV infection. comparison with liver biopsy and fibrotest. Hepatology 2007;46:32-36.

18. Lin ZH, Xin YN, Dong QJ, Wang Q, Jiang XJ, Zhan SH, et al. Performance of the aspartate aminotransferase-to-platelet ratio index for the staging of hepatitis C-related fibrosis: an updated meta-analysis. Hepatology 2011;53:726-736.

19. Itenov TS, Bangert K, Christensen PH, Jensen JU, Bestle MH, Procalcitonin and Survival Study (PASS)-study group. Serum and plasma neutrophil gelatinase associated lipocalin (NGAL) levels are not equivalent in patients admitted to intensive care. J Clin Lab Anal 2014;28:163-167.

20. Castillo-Rodriguez E, Fernandez-Prado R, Martin-Cleary C, PizarroSánchez MS, Sanchez-Niño MD, Sanz AB, et al. Kidney injury marker 1 and neutrophil gelatinase-associated lipocalin in chronic kidney disease. Nephron 2017;136:263-267. 
21. Holzscheiter L, Beck C, Rutz S, Manuilova E, Domke I, Guder WG, et al. NGAL, L-FABP, and KIM-1 in comparison to established markers of renal dysfunction. Clin Chem Lab Med 2014;52:537-546.

22. Bolignano D, Coppolino G, Lacquaniti A, Nicocia G, Buemi M. Pathological and prognostic value of urinary neutrophil gelatinase-associated lipocalin in macroproteinuric patients with worsening renal function. Kidney Blood Press Res 2008;31:274-279.

23. Moledina DG, Hall IE, Thiessen-Philbrook H, Reese PP, Weng FL, Schröppel $B$, et al. Performance of serum creatinine and kidney injury biomarkers for diagnosing histologic acute tubular injury. Am J Kidney Dis 2017;70:807-816.

24. Choudhary NS, Kumar A, Bodh V, Bansal SB, Sharma R, Jain M, et al. Efficacy and safety of sofosbuvir-based regimens in chronic hepatitis C patients on dialysis. Indian J Gastroenterol 2017;36:113-116.

25. Li T, Qu Y, Guo Y, Wang Y, Wang L. Efficacy and safety of direct-acting antivirals-based antiviral therapies for hepatitis $C$ virus patients with stage 4-5 chronic kidney disease: a meta-analysis. Liver Int 2017;37:974-981.

26. Grubb A. Non-invasive estimation of glomerular filtration rate (GFR). The Lund model: simultaneous use of cystatin C- and creatinine-based GFR-prediction equations, clinical data and an internal quality check. Scand J Clin Lab Invest 2010;70:65-70.
27. Ralib AM, Nanyan S, Mat Nor MB. Dynamic changes of plasma neutrophil gelatinase-associated lipocalin predicted mortality in critically ill patients with systemic inflammatory response syndrome. Indian J Crit Care Med 2017;21:23-29.

28. Choi JW, Fujii T, Fujii N. Elevated plasma neutrophil gelatinase-associated lipocalin level as a risk factor for anemia in patients with systemic inflammation. Biomed Res Int 2016;2016:9195219.

29. Sansonno D, Lauletta G, Montrone M, Grandaliano G, Schena FP, Dammacco $F$. Hepatitis $C$ virus RNA and core protein in kidney glomerular and tubular structures isolated with laser capture microdissection. Clin Exp Immunol 2005;140:498-506.

30. Goossens N, Hoshida Y. Hepatitis C virus-induced hepatocellular carcinoma. Clin Mol Hepatol 2015;21:105-114.

31. Villani R, Facciorusso A, Bellanti F, Tamborra R, Piscazzi A, Landriscina $M$, et al. DAAs rapidly reduce inflammation but increase serum VEGF Level: a rationale for tumor risk during anti-HCV treatment._PloS One 2016;11:e0167934.

32. Carrier P, Essig M, Debette-Gratien M, Sautereau D, Rousseau A, Marquet $P$, et al. Anti-hepatitis $C$ virus drugs and kidney. World J Hepatol 2016;8:1343-1353. 